\title{
Local scale fracture characterization of an advanced structured material manufactured by fused deposition modeling in $3 \mathrm{D}$ printing
}

Joseph Marae Djouda

ERMESS, EPF-Engineering school, 3 bis Rue Lakanal, 92330 Sceaux, France

Centre des Matériaux, MINES ParisTech, CNRS UMR 7633, BP 87, 91003, Evry, France

joseph.marae_djouda@epf.fr

Donato Gallittelli, Marouene Zouaoui, Ali Makke, Julien Gardan

ERMESS, EPF-Engineering school, 2 rue Fernand Sastre, Troyes, France

donato.gallitelli@gmail.com

marouene.zouaoui@utt.fr

almak_21@hotmail.com

Julien.Gardan@epf.fr

Naman Recho

ERMESS, EPF-Engineering school, 3 bis Rue Lakanal, 92330 Sceaux, France

Université Clermont Awvergne, Institut Pascal CNRS-UMR 6602, PB 10448, 63000 Clermont-Ferrand, France.

Naman.Recho@epf.fr

Jérôme Crépin

Centre des Matériaux, MINES ParisTech, CNRS UMR 7633, BP 87, 91003, Evry, France

jerome.crepin@mines-paristech.fr

\begin{abstract}
.
Additive manufacturing (AM) is a promising way to produce complex structures by adding layers. AM arises great interest both in industrial and academic sectors to develop new advanced structured materials. To benefit from its advantages, it is important to accurately characterize the mechanical properties of the obtained structures in order to ensure their integrity during operation. It becomes important to characterize these structures at the local scale (micron and/or the nanometer scale). In the specific case of polymeric materials produced by Fused Deposition Modeling (FDM), the comprehension of the mechanical behavior between adjacent layers during deformation can help to improve mechanical properties. However, few studies in the literature have focused on implementing approaches to characterize local strains at the surface of these materials. In this study, an original approach based on the use of a speckle pattern with particles average
\end{abstract}

\section{OPEN ACCESS}

Citation: Djouda, J. M., Gallitelli, D., Zouaoui, M., Makke, A., Gardan, J., Recho, N., Crépin, J., Local scale fracture characterization of an advanced structured material manufactured by fused deposition modeling in 3D printing, Frattura ed Integrità Strutturale, 51 (2021) 534-540.

Received: 31.10 .2019

Accepted: 06.12.2019

Published: 01.01.2020

Copyright: (C) 2020 This is an open access article under the terms of the CC-BY 4.0, which permits unrestricted use, distribution, and reproduction in any medium, provided the original author and source are credited. 
size of 20 microns in diameter was coupled to digital image correlation (DIC). It has been applied to the case of a SENT structure with a notch made by FDM. The successive images recorded by a digital microscope allow a qualitative analysis of the evolutions of the local strains. The kinematic fields are obtained by DIC. The strain evolutions at the notch's tip are highlighted. The deformation mechanisms at the local scale are confronted with the macroscopic behavior of the structure.

KEYWORDS. Additive manufacturing; 3D printing; Digital Image Correlation; Micro speckle; Fracture mechanics.

\section{INTRODUCTION}

A dditive manufacturing (AM) is a promising principle able to shape complex structures through a layer by layer manufacturing. In recent years, AM has been the subject of several developments both by academic and/or industrial researchers. AM has great potential for the manufacture of several structures that can be integrated into the industrial sectors such as aeronautics, automotive and others [1]. To benefit from its advantages, it is important to characterize accurately the obtained structures to allow a better integration in industrial applications. In the specific case of fused deposition modeling (FDM) technologies, the mechanical behavior of the obtained structures suffers from anisotropy, which is introduced by the weld lines and the air gap in the printed part [2]. Many studies aiming to improve the mechanical properties of these structures have been conducted. Some of these studies focus on the modification of the shape of the printed patterns [3] and others are based on improving 3D printing algorithms to enhance the local fracture toughness of printed thermoplastics [4]. Besides of all these processes of pre-structured material depositions, accurate characterization of the mechanical behaviour of the obtained structure is determinant. The knowledge of their mechanical properties is important for use in the technological application. The mechanical characterization of a material goes through a knowledge of its properties both at macroscopic and local scales.

Many technics have been developed to analyze strain evolutions at the local scale. These technics are based on the used periodic or random gratings and have been applied in most of the case in metal specimens [5]-[10]. The periodic gratings consist in arrays of cross lines or of nanoparticles and are deposited by lithographic technics (electron beam lithography, holographic or photolithography). The periodic gratings can be coupled with the technics like Moiré Interferometry [11] or with a specific approach like the one developed in our previous studies [8], [12].

The technics very often associated with the random gratings are Moiré interferometry and digital image correlation (DIC) [13]. In these technics, the roughness of the samples can be used in certain cases. In other cases, paint speckle pattern, deposition and annealing of thin metal layer in order to obtain metal nanoparticles, and lithography technics can be used to deposit gratings [6]. In a very recent study, DIC has been used to measure strains at the surface of 3D printed structures with a large dimension [14].

The DIC analyses the grey levels between two images, one of which is considered as "reference" and the other is "deformed". In this technic, the images used can be obtained by various imaging systems: optical or digital microscopes, scanning electron microscope, atomic force microscope, and others. In addition to the surface kinematic fields, DIC could allow to access the out-of-plane displacements. In the basic version of DIC, the roughness of the surface can be used like a speckle pattern but sometime the contrast of images is not enough. Simple surface preparation may be appropriate depending on material (polymers or alloys) and imaging technique used [5], [6].

The spatial resolution of strains measured by technics using gratings is strongly related to the grating parameters: the size of particles and pitch. With the progress in nanotechnology and nanofabrication, it is possible to deposit gratings with dozens of nanometre of pitch and diameter of nanoparticles.

However, it is difficult to use lithographic techniques to deposit gratings on the surface of a polymer structure, because of the solutions used during the nanofabrication process. Moreover, it could be straightforward to image polymer materials by SEM. The key challenge for better understanding mechanisms leading to structure damages or failures remains the development of tools giving direct access to the local scale material behaviour. For a good characterization of polymer structures obtained by AM, it is necessary to develop new techniques to measure local deformations. 
In this study, an original process is developed to analyze strain evolutions at the surface of polymers structures obtained by AM. It consists in deposing speckle pattern with micron dimension. An in situ tensile test under a digital microscope in which images of the specimen surface are acquired is conducted until the specimen failure. The images obtained are analyzed with DIC tools. The specimen consists in a mini SENT. The deformation evolutions are analyzed for different loading levels and local mechanisms of deformation are highlighted. The local strain evolutions are confronted with the macroscopic behavior of the specimen.

\section{METHODS AND RESULTS}

$\mathrm{I}$ $\mathrm{n}$ this section, the detail of specimens' preparation and the implementation of tests are presented. Moreover, the main results will be discussed.

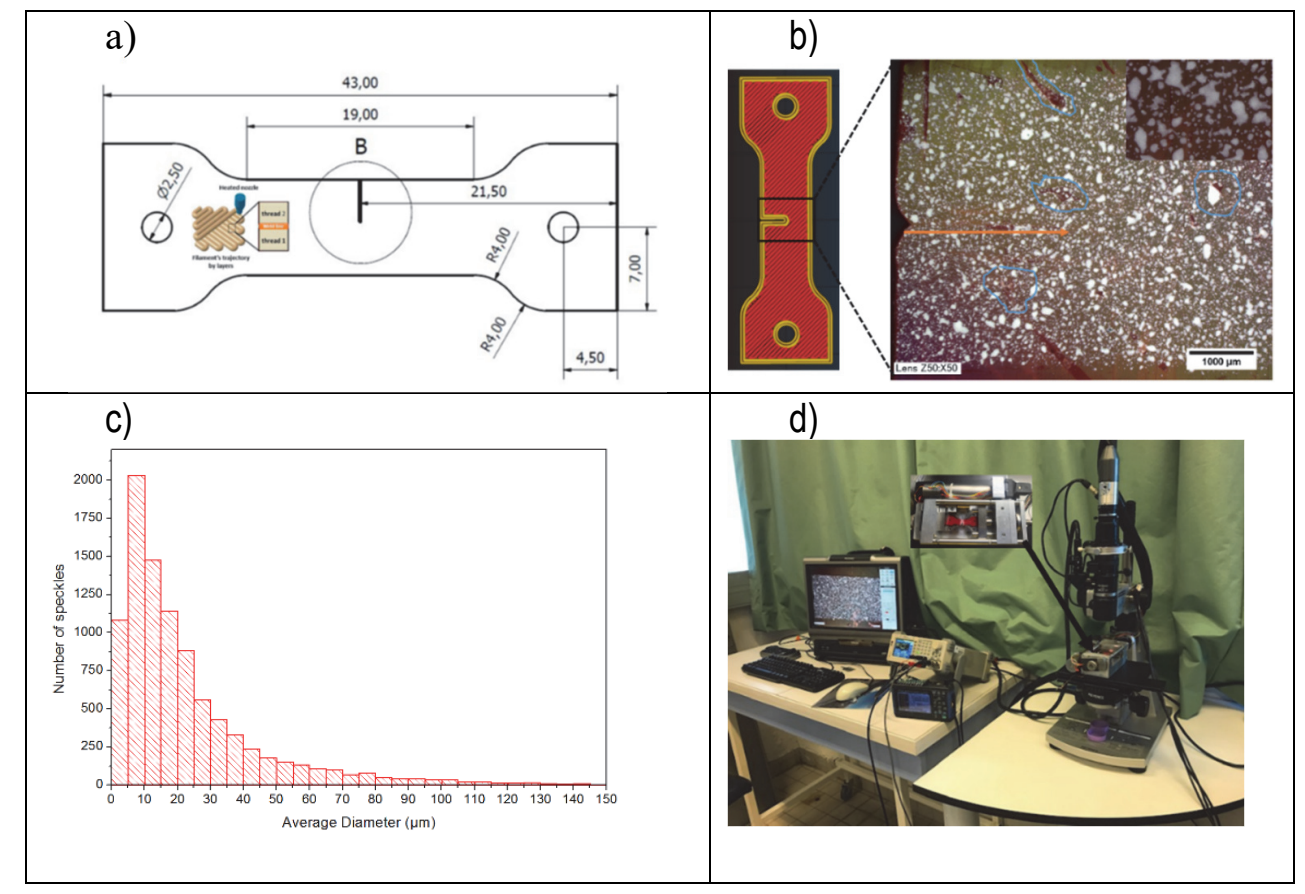

Figure 1: a) Plan of the sample used in the study. It consists to SENT specimen with a notch set as $a_{0} / w=0.45$. b) The sample is $3 \mathrm{D}$ printed layer by layer and oriented $+45^{\circ} /-45^{\circ}$. After surface preparation of the sample, the micro-speckle pattern is deposited. c) Histogram shows the diameter distribution of speckles at the sample surface. More than $70 \%$ of speckles have their diameter less than $20 \mu \mathrm{m} . \mathrm{d}$ ) The experimental set up consist in a digital microscope for the surface observation, a tensile micromachine and trigger system.

\section{Specimen preparation}

The specimen is printed by adding melted layers of Acrylonitrile butadiene styrene (ABS) using a makerbot replicator $2 \mathrm{X}$. The layers were deposed at a temperature of $235^{\circ} \mathrm{C}$ with a $+45^{\circ} /-45^{\circ}$ oriented filaments as described in Fig. 1a). A layer thickness of $0.25 \mathrm{~mm}$ was used except for the first one $(0.3 \mathrm{~mm})$ in order to ensure good adhesion with the manufacturing tray. A mini SENT specimen is 3D printed with the dimension showed in Fig. 1a). The notch was also made by AM. However, because of the temperature during the manufacturing, the layers have closed at the notch and the latter is not clearly visible. The yellow arrow in Fig. 1-b) illustrates its position on the specimen surface. The notch was made to meet the condition $a_{0} / w=0.45$ as recommend in ASTM E1820 standard; $a_{0}$ represents the initial length of the notch and $w$ the width at the useful part of specimen. It is important to notice that the specimen like design herein is not standardized. The initial thickness of the specimen 3D printed was of $6 \mathrm{~mm}$ and by mechanical polishing each side, the final thickness considered was $3 \mathrm{~mm}$ in order to reduce the influence of surface defects on the mechanical feature. The final surface of the specimen and also to obtain a soft surface in order to deposit the speckle pattern to allow the use of the DIC. Some defects (hole, void ...) are visible on the specimen surface. 

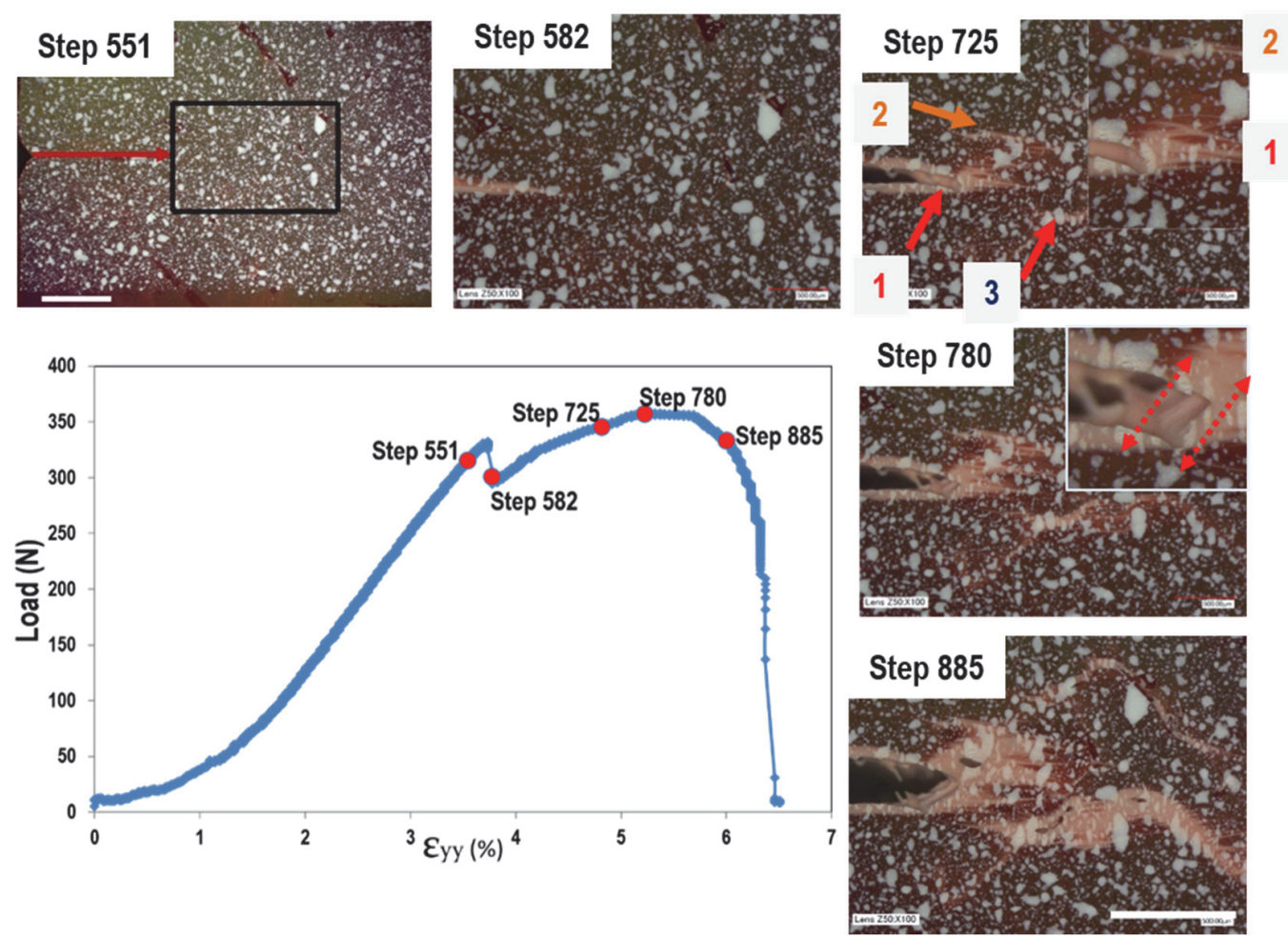

Figure 2: Strain evolutions from the optical images. The image at step 551 is recorded at the magnification $x 50$, the notch is not visible at this loading level. The applied load leads to the sudden opening of the notch. The images recorded after the notch opening is at the magnification x100 to allow a better visualisation of the local strain evolutions. The scale bar is $1 \mathrm{~mm}$ in all the images.
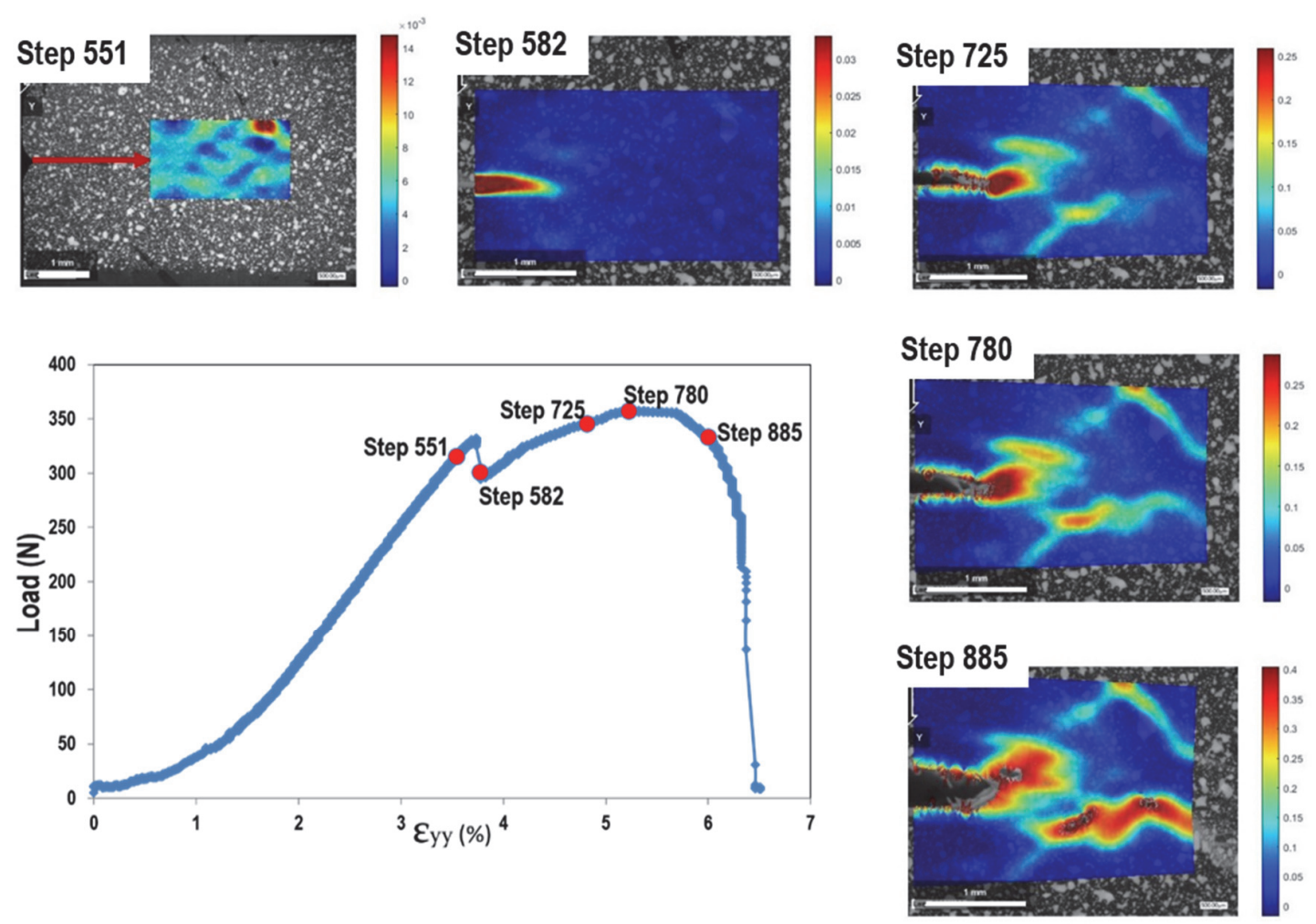

Figure 3: Quantitative analysis of strain evolutions. Step 551 shows the region where the strains are analyzed in front of the design notch materialized by the red arrow. The scale bar at each image is $1 \mathrm{~mm}$. 


\section{Speckel characterization}

DIC consists in a segmentation of an image into subsets. The changes in the different subsets on the successive images are followed. The dimensions of the speckles and their number in each subset are determinant to obtain a good resolution. In this study, an original speckle pattern with micron dimensions is deposited. In contrary of the conventional process of speckle pattern deposition with the white base coat, in the present study the surface of the sample (red) was used as the base. White speckles of micrometric dimensions were deposited using an airbrush. This results in a speckle pattern with the particles of 20 microns of average diameter (see Fig. 1-c)).

\section{In situ tensile test}

Once the speckle grating deposited at the surface of the sample, an in situ tensile test under a digital microscope was conducted. The experimental set up consists in a numerical microscope Keyence VHX-1000 made by Keyence Corporation TSE for the surface observation, a tensile micro-machine and a trigger system (see Fig. 1-d)). The trigger system allows recording of images at a specific rate when the specimen is continuously solicited during a tensile test and related each image to the corresponding applied load in the macroscopic behavior of the specimen. One image was recorded per second during the test until the failure of the specimen.

\section{DISCUSSION OF THE RESULTS}

7 he process developed herein allows obtaining both macroscopic and local evolutions of the deformations of the material. Fig. 2 shows the strain - load curve. At the elastic-plastic transition (around 3.6\% of macroscopic deformation), an important decrease of the applied load is observed, and this materializes the sudden opening of the notch. This last is initially enclosed due to the deposition temperature of neighboring filaments during the deposition process. After this load variation, the applied load increases again, reach to one threshold, and decrease until the failure of the specimen. Some images are shown in Fig. 2 in order to illustrate the main local features at the specimen surface.

The applied load leads to a gradual deformation at the tip of the crack. Once the crack reaches the design length $a_{0}$, the concentric curved filaments at the crack tip begin to experience deformation. The deformation at the crack tip corresponds to the strain lobe visible in Fig. 2 - Step 725). Another's strain concentration zones (zones (2) and (3)) are also visible. The zone (1) corresponds to the crack tip, zones (2) and (3) are equidistant from the perpendicular to the direction of traction passing through the initial crack direction. The zones (2) and (3) could correspond to the boundaries between the two concentric curved filaments materializing the crack tip and layers oriented $+45^{\circ} /-45^{\circ}$ of the specimen (see complementary material). However, it is important to notice that the positions of strain initiation at the surface of the specimen do not correspond to visible defects (hole, void). They could correspond to defects in the volume of the material, to filling defects between curved filaments and oriented layers $+45^{\circ} /-45^{\circ}$. Once the crack has spread and is beyond the two curved filaments, layers oriented $+45^{\circ} /-45^{\circ}$ begins to deform. A V-shape notch tip is observed at the crack tip and the deformation of the layers oriented $+45^{\circ} /-45^{\circ}$ highlighted tears marks oriented $+45^{\circ}$. The strain marks between the crack tip and zone (2) increase gradually until they overlap (See Fig. 2 - Step 780). With the applied load, zone (1) and zone (2) form now a unique zone of strain concentration, the strain at the zone (3) have also greatly increased and the cavities developed in the volume of the material are visible at the surface.

Ncorr software was used for the strain quantitative analysis. Ncorr is an open-source 2D-DIC MATLAB Software developed at Georgia Institute of Technology. Furthermore, this software integrated the modern algorithm of DIC [15]. Fig. 3 presents the strain maps corresponding to images from the previous section. The strain $\varepsilon_{y y}$ was computed using the Green-Lagrangian formalism in which the four-displacement gradient is used in order to reduce noise from differentiation. The strain concentration and magnification are in good agreement with the deformation evolutions observed. At step 551, no strain concentration is visible at the surface of the specimen. This step is macroscopically located in the elastic domain of the material. The plastic domain is accompanied by the sudden opening of the notch. The strain map of step 582 shows clearly strain concentrations at the crack tip. These strains could correspond to the beginning of deformation of the curved filaments as observed in the images. Another strain concentration zone (zone (2)) is visible. The strains are quite uniform in the rest of the specimen.

By increasing the applied load, strain at the crack tip increases in dimension and in magnification (Fig. 3 - Step 725). Locally strain is around $25 \%$ and macroscopic strain value is less than $5 \%$. Then local strain can reach very significant values compared to macroscopic one. Similar observations were made in the case of austenitic stainless steel 316L by applying the nanogauges technic [7]. It is important to notice that strain at the crack tip grows in the direction of the zone (2). Another 
observation is the strain concentration materializing the zone (3) like indicated in the previous section. The beginning of this zone below to the crack could also correspond to the frontier between the curved filaments and filaments oriented $45^{\circ}$. At step 780, strain at the crack has increased so that they start to overlap with those of the zone (2). The strain at the zone (3) have also increased and at the different strain concentration area, the strain magnification is up than $40 \%$. Step 780 is macroscopically at the beginning of one plateau. This last could express the competition between cavities growth and necking.

At step 885, the crack tip and zone (2) overlap and form a unique strain concentration zone. This unique zone contains the crack notch and with the zone (3), they now form the two mains strain concentration zones. They are all oriented almost perpendicular to the tensile direction and the crack tip seems to interact with the zone (3). The local strain intensity reaches the values up to $100 \%$. Macroscopically, this step is in the necking domain, a few moments before the failure of the specimen.

\section{CONCLUSIONS}

$\mathrm{I}$ $\mathrm{n}$ this study, an original approach is developed for local strain characterization at the surface of polymer materials produced by Fused Depositing Modeling (FDM) in 3D printing. This approach allows a comprehensive qualitative analysis of the local deformation from the optical image recorded during the in-situ tensile test. The micro speckle pattern deposited at the surface of the specimen ensures the strain quantification from a DIC software. Ncorr 2D-DIC MATLAB Software was used. The strain evolutions in the strain maps are in good agreement with the strain evolutions observed in the optical images. The surface strain evolutions are quantified and the interactions between strain concentration zones are clearly evidenced. These strain concentration zones could correspond to filling defects between curved filaments and oriented layers $+45^{\circ} /-45^{\circ}$. The local strain values are very important compared to the macroscopic ones and the gap increases with the applied load. The approach developed herein presents great potential for local characterization of materials in general and for polymer materials specifically because of the limitations they present in regard of the classical approach. It also brings precious details which could help for the optimization of smart materials or pre-structured materials by additive manufacturing.

\section{REFERENCES}

[1] J. Gardan, “Additive manufacturing technologies: State of the art and trends," Int. J. Prod. Res., vol. 7543, no. November, pp. 149-168, 2015.

[2] S. H. Ahn, M. Montero, D. Odell, S. Roundy, and P. K. Wright, Anisotropic material properties of fused deposition modeling $A B S$, vol. 8, no. 4. 2002.

[3] D. Prall and R. S. Lakes, "chiral honeycombe. Poisson's ratio. 1. INTRODUCTION Cellular solids are used widely in a variety of engineering applications. In particular," Int. J. Mech. Sci., vol. 39, no. 3, 1997.

[4] J. Gardan, A. Makke, and N. Recho, "Improving the fracture toughness of 3D printed thermoplastic polymers by fused deposition modeling," Int. J. Fract., vol. 210, no. 1-2, pp. 1-15, 2018.

[5] L. Allais, M. Bornert, T. Bretheau, and D. Caldemaison, "Experimental characterization of the local strain field in a heterogeneous elastoplastic material," Acta Metall. Mater., vol. 42, no. 11, pp. 3865-3880, 1994.

[6] S.-H. Joo, J. K. Lee, J.-M. Koo, S. Lee, D.-W. Suh, and H. S. Kim, “Method for measuring nanoscale local strain in a dual phase steel using digital image correlation with nanodot patterns," Scr. Mater., vol. 68, no. 5, pp. 245-248, Mar. 2013.

[7] J. Marae Djouda, G. Montay, B. Panicaud, J. Béal, Y. Madi, and T. Maurer, "Nanogauges gratings for strain determination at nanoscale," Mech. Mater., vol. 114, 2017.

[8] J. Marae Djouda et al., "Investigation of Nanoscale Strains at the Austenitic stainless steel 316L surface : Coupling between Nanogauges Gratings and EBSD Technique during in situ Tensile Test .," Mater. Sci. Eng. A, vol. 740-741, no. July 2018, p. 316, 2018.

[9] R. Moulart, R. Rotinat, F. Pierron, and G. Lerondel, "On the realization of microscopic grids for local strain measurement by direct interferometric photolithography," Opt. Lasers Eng., vol. 45, no. 12, pp. 1131-1147, Dec. 2007.

[10] A. Clair et al., "Strain mapping near a triple junction in strained Ni-based alloy using EBSD and biaxial nanogauges," Acta Mater., vol. 59, no. 8, pp. 3116-3123, May 2011.

[11] H. Du et al., "Large-deformation analysis in microscopic area using micro-moiré methods with a focused ion beam 
milling grating," Opt. Lasers Eng., vol. 45, no. 12, pp. 1157-1169, Dec. 2007.

[12] T. Maurer et al., "The beginnings of plasmomechanics: towards plasmonic strain sensors," Front. Mater. Sci., vol. 9, no. 2, pp. 170-177, 2015.

[13] Y. Tanaka, K. Naito, S. Kishimoto, and Y. Kagawa, "Development of a pattern to measure multiscale deformation and strain distribution via in situ FE-SEM observations.," Nanotechnology, vol. 22, p. 115704, 2011.

[14] K. Schnittker et al., "Integrating digital image correlation in mechanical testing for the materials characterization of big area additive manufacturing feedstock," vol. 26, no. December 2018, pp. 129-137, 2019.

[15]J. Blaber, B. Adair, and A. Antoniou, "Ncorr: Open-Source 2D Digital Image Correlation Matlab Software," Exp. Mech., vol. 55, no. 6, pp. 1105-1122, 2015.

\section{COMPLEMENTARY MATERIAL}

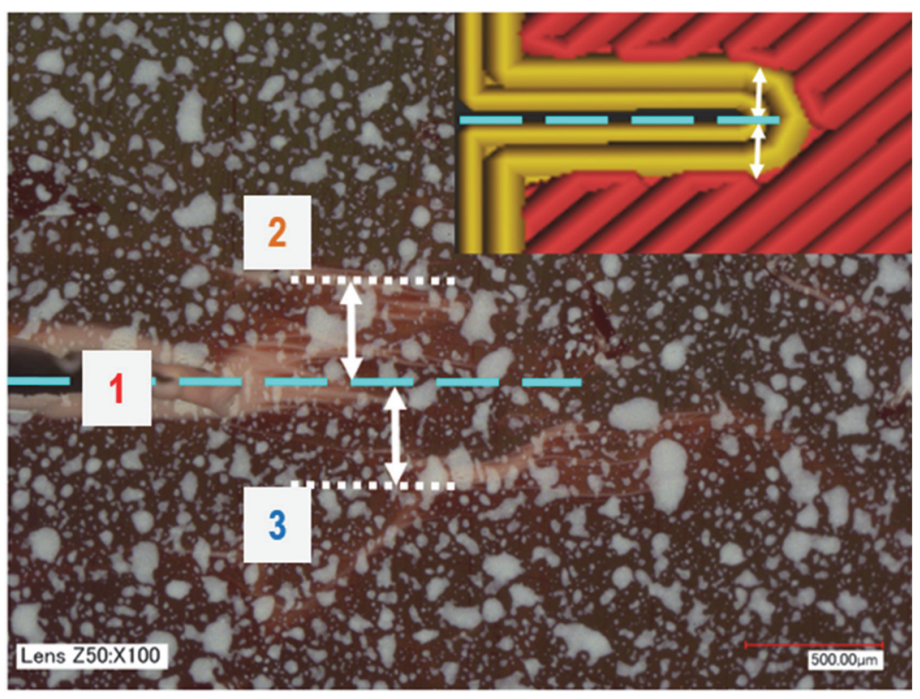

Figure C1: Illustration of the position of zones (2) and (3) in regard of the crack tip (zone (1)) direction. 